Submitted to the Annals of Statistics

arXiv: arXiv: 0000.0000

\title{
SUPPLEMENT TO ASYMPTOTIC THEORY OF CEPSTRAL RANDOM FIELDS
}

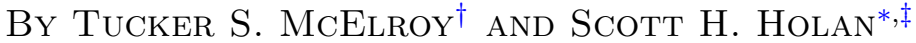 \\ U.S. Census Bureau ${ }^{\dagger}$ and University of Missouri ${ }^{\ddagger}$
}

This supplement contains a discussion of extensions and applications of the basic modeling methodology, as well as all proofs of technical results.

\section{APPENDIX A: EXTENSIONS AND APPLICATIONS}

A.1. Extensions. Extensions to missing data, imputation, and signal extraction are possible, as discussed below. Suppose that there is missing data in our sample of the field. We can always express this situation as $\mathcal{Y}=K Y$, where $\mathcal{Y}$ is now our observed data vector of dimension $N^{2}-T$, where $T$ is the number of missing observations in the field, and $Y$ is the vectorized random field, as described in the article. $K$ is a selection matrix with $N^{2}-T$ rows and $N^{2}$ columns, which eliminates any entries of $Y$ (corresponding to omitted observations in $\mathbb{Y}$ ) for which no measurements exists. Then the covariance matrix of $\mathcal{Y}$ is $K \Sigma(\widetilde{F}) K^{\prime}$, which is easily computed from any given parameter specification. The quadratic form in our Gaussian likelihood is then $(\mathcal{Y}-K \mu)^{\prime}\left[K \Sigma(\widetilde{F}) K^{\prime}\right]^{-1}(\mathcal{Y}-$ $K \mu$ ); a matrix of this form is always invertible. It is obtained by crossing out rows and corresponding columns of $\Sigma(\widetilde{F})$, and contracting the result.

Signal extraction for random fields can be handled as follows. We suppose that our random field $\mathbb{Y}$ consists additively of signal and noise, i.e.,

$$
\mathbb{Y}=\mathbb{S}+\mathbb{N}
$$

The signal random field $\mathbb{S}$ and noise random field $\mathbb{N}$ may each be given by cepstral field models, but are assumed to be completely independent of one another. Each may also have their own regression mean effects. Let the cepstral coefficient grids of each be denoted $\Theta_{\mathbb{S}}$ and $\Theta_{\mathbb{N}}$. We can compute the acf for each component via the methods outlined in Section 2 of the main article, obtaining $\gamma_{\mathbb{S}}$ and $\gamma_{\mathbb{N}}$. In turn we can determine the vectorized versions of signal and noise, denoted by $S$ and $N$, with covariance matrices $\Sigma_{S}$ and $\Sigma_{N}$. With $Y=S+N$ equal to the vectorized data, the quadratic form in the Gaussian likelihood is $(Y-\mu)^{\prime}\left(\Sigma_{S}+\Sigma_{N}\right)^{-1}(Y-\mu)$. So this is easy to calculate given the parameters $\Theta_{\mathbb{S}}$ and $\Theta_{\mathbb{N}}$. The distribution of the signal conditional on the data is Gaussian with mean and variance given by

$$
\mathbb{E}[S]+\Sigma_{S}\left(\Sigma_{S}+\Sigma_{N}\right)^{-1}(Y-\mu) \quad \text { and } \quad \Sigma_{S}-\Sigma_{S}\left(\Sigma_{S}+\Sigma_{N}\right)^{-1} \Sigma_{S}
$$

Of course, the conditional mean is for the vectorized field $S$; in order to get our estimate of $\mathbb{S}$, we reverse the vectorization mapping. Also, if there is missing data and $\mathcal{Y}=K Y$ is our observation vector, the likelihood uses the quadratic form $(\mathcal{Y}-K \mu)^{\prime}\left(K \Sigma_{S} K^{\prime}+K \Sigma_{N} K^{\prime}\right)^{-1}(\mathcal{Y}-K \mu)$, and the conditional mean will now be

$$
K \mathbb{E}[S]+\Sigma_{S} K^{\prime}\left(K \Sigma_{S} K^{\prime}+K \Sigma_{N} K^{\prime}\right)^{-1}(\mathcal{Y}-K \mu) .
$$

\footnotetext{
${ }^{*}$ Supported by NSF and the U.S. Census Bureau under NSF grant SES-1132031, through the NSF-Census Research Network (NCRN) program and by a University of Missouri Research Board grant.

MSC 2010 subject classifications: Primary 62F12, 62M30; Secondary 62F15, 62M10

Keywords and phrases: Bayesian estimation, Cepstrum, Exponential spectral representation, Lattice data, Spatial statistics, Spectral density.
} 
The mean $\mathbb{E}[S]$ is determined in practice by assigning some of the fixed effects in $\widetilde{X}$ to the signal. For example, if the signal is defined to be a smooth "trend" effect and the noise is idiosyncratic noise, then all mean effects might be assigned to the signal so that $\mathbb{E}[S]=\mu$.

A.2. Straw Yield Data. To illustrate the utility of our approach, we analyzed the Mercer and Hall straw yield dataset [12] considered by Solo [14], among others. This dataset can be obtained through the $\mathrm{R}$ contributed package agridat [16]. One important distinction between our analysis and the analysis of Solo [14] is that the lattice in Solo [14] is taken as $19 \times 25$, whereas, similar to Cressie [2] and the dataset as provided in the $\mathrm{R}$ contributed package agridat, our lattice is $20 \times 25$. The reason for the smaller lattice in Solo [14] remains unclear. Also, similar to Cressie [2], we considered the data as aggregations over $3.30 \times 2.51$ meter plots. The observed data are displayed in Figure 1 and comprehensive details of the experiment, as it relates to yields of wheat grain, can be found in Cressie [2].

Exploratory analyses consisting of estimating linear models with independent errors revealed potentially significant "row" and "column" effects. Unlike the model formulation of Solo [14], these trend effects could be readily accounted for within our modeling framework and their statistical significance evaluated. Additionally, further exploratory analysis using the empirical variogram seemed to indicate a negligible nugget effect (measurement error). Therefore, we considered an analysis with no nugget effect and note that if one had an empirical estimate of this effect, it could be easily incorporated into our model. An initial assessment of the spatial correlation was conducted using Moran's $I$ statistic with a first-order nearest neighbor weighting matrix. The $p$ value for testing the null hypothesis of the absence of spatial autocorrelation was $2.2 \times 10^{-16}$, indicating a high-degree of spatial autocorrelation.

There were two main goals of our analysis. First, we wanted to compare our analysis with the previous analysis of Solo [14], as well as illustrate that our methodology can model apparent spatial correlation in the data. Second, we demonstrated that there is a significant row and column effect (trend), and consider models that account for these effects using regressors. We considered several models in our analysis, including models with trend (i.e., row and column effects) removed and not removed. To remove the trend, we regressed the vectorized responses on a design matrix, $X$, consisting of a column of ones followed by columns containing the associated row and column indices, respectively. In the case where no trend was removed, we included a constant mean. Lastly, we considered models in which no trend was removed and no constant-mean was included. Not surprisingly, the latter model performed poorly relative to the other models considered and, therefore, is not discussed further.

Similar to the simulation study in Section 5 of the article, the model autocovariances were calculated according (2.5) of the article, with $M=1000$. Here, we evaluated models with $p_{1}=p_{2}$ equal $1,2,3$. Due to the mirror reflectional symmetry, the models with $p_{1}=1$ had 6 parameters when no trend was removed and 8 parameters when the trend was accounted for. Similarly, for $p_{1}=2$ and $p_{1}=3$ the no-trend model had 14 and 26 parameters, while the model accounting for the trend had 16 and 28 parameters, respectively. Higher-order models could be could considered; however, $p_{1}=2,3$ were sufficient in this context in terms of removing the spatial correlation.

Model selection was conducted using Akaike information criterion (AIC), Bayesian information criterion (BIC), and the Hannan-Quinn information criterion (HQ), defined as $2 k-2 \log (L)$, $k \log \left(N^{2}\right)-2 \log (L)$, and $2 k \log \left\{\log \left(N^{2}\right)\right\}-2 \log (L)$, respectively, where $k, N^{2}=500$, and $L$ denote the number of model parameters, the sample size, and the likelihood function. For the trend and no-trend models, the values of $-\log (L)$, AIC, BIC, and HQ are given in Table 1 . Using the information criteria, it appeared that a model with $p_{1}=2$ or $p_{1}=3$, with the trend accounted for, was preferred. For reasons of model parsimony and because AIC is known to over-fit, we chose the 
$p_{1}=2$ model, with the trend accounted for.

Table 2 provides the estimated model parameters along with their standard errors (as determined by the square root of the diagonal elements of the inverse observed Hessian matrix). Judging by the $95 \%$ confidence intervals for the estimated parameters (e.g., $\widehat{\theta} \pm 2 \times \operatorname{se}(\widehat{\theta})$ and $\widehat{\beta} \pm 2 \times \operatorname{se}(\widehat{\beta})$ ) the trend parameters, $\beta$, are clearly statistically significant. Applying Moran's $I$ statistic with a first-order nearest neighbor weighting matrix to the residuals (i.e., $\varepsilon=\Sigma\left(F_{\widehat{\theta}}\right)^{-1 / 2}(Y-\mu)$ ) yielded a $p$-value of 0.999 , which indicates that the spatial autocorrelation has been removed.

It should be noted that fitting a model without accounting for trend (i.e., constant mean only; $p_{1}=2$ ) also removes the spatial autocorrelation (Moran's $I$ statistic has $p$-value $=1$ ). However, since the row and column effects are clearly statistically significant and the model having the trend accounted for is preferred according to the information criteria, we ultimately selected the $p_{1}=2$ trend model. It is also of worth noting that the mean parameters, as specified here, do not suffer from the "spatial confounding" problem recently described in the spatial statistics literature; e.g., see Hodges and Reich [6], Paciorek [13], and the references therein. This lack of spatial confounding is clearly illustrated in Section 5 of the article, where we can accurately estimate the true trend parameters. Finally, the approach proposed herein provides a natural framework for inclusion of trend parameters within the model, which is not the case with the models proposed in Solo [14]. Even in the constant mean case, the approach of Solo [14] would need to estimate the mean nonparametrically (e.g., using the sample mean) and remove it prior to model estimation.

\section{APPENDIX B: PROOFS}

Derivation of (2.5) in the article. First we calculate $\left\{\bar{E}^{\prime}[\Theta] E\right\}_{s_{1}, s_{2}}$, which equals

$$
\begin{aligned}
& \sum_{k_{1}, k_{2}=1}^{2 p_{1}+1} e^{\left\{-i \pi \frac{\left(p_{1}+1-k_{1}\right)\left(M+1-s_{1}\right)}{M}\right\}} \Theta_{k_{2}-p_{1}-1, p_{1}+1-k_{1}} e^{\left\{i \pi \frac{\left(p_{1}+1-k_{2}\right)\left(M+1-s_{2}\right)}{M}\right\}} \\
& =\sum_{j_{1}, j_{2}=-p_{1}}^{p_{1}} \Theta_{j_{1}, j_{2}}\left[\exp \left\{-i \pi \frac{\left(M+1-s_{1}\right)}{M}\right\}\right]^{j_{1}}\left[\exp \left\{-i \pi \frac{\left(M+1-s_{2}\right)}{M}\right\}\right]^{j_{2}} .
\end{aligned}
$$

Then using equation (2.3) of the article, we obtain

$$
\log F\left(\pi \frac{M+1-s_{2}}{M}, \pi \frac{M+1-s_{1}}{M}\right)=\left\{\bar{E}^{\prime}[\Theta] E\right\}_{s_{1}, s_{2}}
$$

for $1 \leq s_{1}, s_{2} \leq 2 M+1$. So we can evaluate $F$ on the desired grid of frequencies by exponentiating each entry of the matrix $\bar{E}^{\prime}[\Theta] E$. Discretizing equation (2.2) of the article yields

$$
\begin{aligned}
\gamma_{h_{1}, h_{2}} \approx & (2 M+1)^{-2} \sum_{s_{1}, s_{2}=1}^{2 M+1} F\left(\pi \frac{M+1-s_{2}}{M}, \pi \frac{M+1-s_{1}}{M}\right) \\
& \cdot \exp \left\{i \pi h_{1} \frac{M+1-s_{2}}{M}\right\} \exp \left\{i \pi h_{2} \frac{M+1-s_{1}}{M}\right\} \\
= & (2 M+1)^{-2} \sum_{s_{1}, s_{2}=1}^{2 M+1} \exp \left\{\left(\bar{E}^{\prime}[\Theta] E\right)_{s_{1}, s_{2}}\right\} \bar{G}_{h_{1}+H+1, s_{2}} G_{H+1-h_{2}, s_{1}}
\end{aligned}
$$

for $1 \leq j_{1} \leq 2 H+1$ and $1 \leq j_{2} \leq 2 M+1$. Then we can compute $[\Gamma]$ directly as follows:

$$
[\Gamma]_{k_{1}, k_{2}}=\gamma_{k_{2}-H-1, H+1-k_{1}} \approx(2 M+1)^{-2} \sum_{s_{1}, s_{2}=1}^{2 M+1} \exp \left\{\left(\bar{E}^{\prime}[\Theta] E\right)_{s_{1}, s_{2}}\right\} \bar{G}_{k_{2}, s_{2}} G_{k_{1}, s_{1}} \text {. }
$$


In matrix form this is equation (2.5) of the article.

Proof of Proposition 2.1. First, consider decomposing the cepstral matrix $\Theta$ into four quadrants, plus the four semi-axes, plus the origin (Kizilkaya [8] has a similar decomposition). In the following treatment, we can let $p_{1}=\infty$ for a general decomposition, or take $p_{1}<\infty$ for the purposes of modeling:

$$
\begin{aligned}
\sum_{j_{1}, j_{2}=-p_{1}}^{p_{1}} \Theta_{j_{1}, j_{2}} Z_{1}^{j_{1}} Z_{2}^{j_{2}} & =\Theta_{0,0}+\sum_{j_{1}, j_{2}=1}^{p_{1}} \Theta_{j_{1}, j_{2}} Z_{1}^{j_{1}} Z_{2}^{j_{2}}+\sum_{j_{1}, j_{2}=1}^{p_{1}} \Theta_{j_{1}, j_{2}} Z_{1}^{-j_{1}} Z_{2}^{-j_{2}} \\
& +\sum_{j_{1}, j_{2}=1}^{p_{1}} \Theta_{-j_{1}, j_{2}} Z_{1}^{-j_{1}} Z_{2}^{j_{2}}+\sum_{j_{1}, j_{2}=1}^{p_{1}} \Theta_{-j_{1}, j_{2}} Z_{1}^{j_{1}} Z_{2}^{-j_{2}} \\
& +\sum_{j_{1} \neq 0} \Theta_{j_{1}, 0} Z_{1}^{j_{1}}+\sum_{j_{2} \neq 0} \Theta_{0, j_{2}} Z_{2}^{j_{2}} .
\end{aligned}
$$

We have used some of the simple reflection properties of the cepstral matrix for this decomposition. Also, terms are grouped appropriately, so that they correspond to the causal field, skew field, and the axes' fields as follows:

$$
\begin{aligned}
\sum_{j_{1}, j_{2} \geq 0} \psi_{j_{1}, j_{2}} Z_{1}^{j_{1}} Z_{2}^{j_{2}} & =\exp \left\{\sum_{j_{1}, j_{2}=1}^{p_{1}} \Theta_{j_{1}, j_{2}} Z_{1}^{j_{1}} Z_{2}^{j_{2}}\right\} \\
\sum_{j_{1}, j_{2} \geq 0} \phi_{j_{1}, j_{2}} Z_{1}^{-j_{1}} Z_{2}^{j_{2}} & =\exp \left\{\sum_{j_{1}, j_{2}=1}^{p_{1}} \Theta_{-j_{1}, j_{2}} Z_{1}^{-j_{1}} Z_{2}^{j_{2}}\right\} \\
\sum_{j_{1} \geq 0} \xi_{j_{1}} Z_{1}^{j_{1}} & =\exp \left\{\sum_{j_{1}=1}^{p_{1}} \Theta_{j_{1}, 0} Z_{1}^{j_{1}}\right\} \\
\sum_{j_{2} \geq 0} \omega_{j_{2}} Z_{2}^{j_{2}} & =\exp \left\{\sum_{j_{2}=1}^{p_{1}} \Theta_{0, j_{2}} Z_{2}^{j_{2}}\right\} .
\end{aligned}
$$

By expanding the exponentials as power series on the right hand side of (B.2), we see that $Z_{1}$ and $Z_{2}$ both occur at least once in every term; hence we must have $\psi_{0,0}=1, \psi_{0,1}=0$, and $\psi_{1,0}=0$. The other coefficients $\psi_{j_{1}, j_{2}}$ have no constraints, but are determined by the cepstral coefficients. Similarly $\phi_{0,0}=1, \phi_{0,1}=0$, and $\phi_{1,0}=0$, while $\xi_{0}=1=\omega_{0}$.

Comparing these associations with (B.1), we see at once that the spectrum is equal to the magnitude squared of the product of the above factors (B.2), (B.3), (B.4), and (B.5), along with the constant $e^{\Theta_{0,0}}$. Taking the product of the corresponding squared magnitudes yields

$$
\begin{aligned}
& e^{\Theta_{0,0}} \cdot \sum_{s_{1}, s_{2}, j_{1}, j_{2}, k_{1}, k_{2} \in \mathbb{Z}} \gamma_{s_{1}, s_{2}}(\Psi) \gamma_{j_{1}, j_{2}}(\Phi) \gamma_{k_{1}}(\Xi) \gamma_{k_{2}}(\Omega) Z_{1}^{s_{1}-j_{1}+k_{1}} Z_{2}^{s_{2}+j_{2}+k_{2}} \\
& =e^{\Theta_{0,0}} \cdot \sum_{h_{1}, h_{2} \in \mathbb{Z}}\left(\sum_{j_{1}, j_{2} \in \mathbb{Z}} \gamma_{j_{1}, j_{2}}(\Phi)\left[\sum_{k_{1}, k_{2} \in \mathbb{Z}} \gamma_{h_{1}+j_{1}-k_{1}, h_{2}-j_{2}-k_{2}}(\Psi) \gamma_{k_{1}}(\Xi) \gamma_{k_{2}}(\Omega)\right]\right) Z_{1}^{h_{1}} Z_{2}^{h_{2}} .
\end{aligned}
$$

Matching coefficients (i.e., computing the inverse DFT) then produces equation (2.8) of the article.

In order to obtain equations (2.9) through (2.12) of the article, we must obtain the field coefficients in terms of known cepstral matrix coefficients. Differentiating (B.2) with respect to $Z_{1}$ 
yields

$$
\sum_{j_{1}, j_{2} \geq 0} \psi_{j_{1}, j_{2}} j_{1} Z_{1}^{j_{1}-1} Z_{2}^{j_{2}}=\sum_{j_{1}, j_{2} \geq 1}\left(\sum_{k_{1}, k_{2} \geq 1} \psi_{j_{1}-k_{1}, j_{2}-k_{2}} \Theta_{k_{1}, k_{2}} k_{1}\right) Z_{1}^{j_{1}-1} Z_{2}^{j_{2}}
$$

as a formal expression, after recollecting terms. Then matching terms, we find a recursive relation between $\psi_{j_{1}, j_{2}}$ (where both $j_{1}$ and $j_{2}$ are positive) and $\Theta_{k_{1}, k_{2}}$, given below. Similar calculations for the other associations (B.3), (B.4), and (B.5) yield equations (2.9) through (2.12) of the article.

Derivation of (3.1) in the article. If we suppose that $\mu=\widetilde{X} \beta$ is the true mean of the field, then the sample mean $\gamma_{h_{1}, h_{2}}\left(I_{\beta}\right)$ is given by

$$
\gamma_{h_{1}, h_{2}}\left(I_{\beta}\right)=N^{-2} \sum_{t_{1}=1}^{N_{1}} \sum_{t_{2}=1}^{N_{2}} \mathbb{W}_{t_{1}, t_{2}} \mathbb{W}_{t_{1}+h_{1}, t_{2}+h_{2}}
$$

Substituting this into the expression for KL, we obtain

$$
\mathrm{KL}\left(F_{\theta}, I_{\beta}\right)=\left\langle\log F_{\theta}\right\rangle+N^{-2} \sum_{t_{1}, t_{2}=1}^{N_{1}} \sum_{s_{1}, s_{2}=1}^{N_{2}} W_{N_{2}\left(t_{1}-1\right)+t_{2}} W_{N_{2}\left(s_{1}-1\right)+s_{2}} \gamma_{s_{1}-t_{1}, s_{2}-t_{2}}\left(F_{\theta}^{-1}\right) .
$$

Recalling that $Y_{t}-\mu_{t}=W_{t}$ and the definition of $\Sigma\left(F_{\theta}^{-1}\right)$, we obtain (3.1) of the article from (B.6).

Proof of Lemma 4.1. For notational convenience in the proofs, we will consider the frequencies in the interval $[0,2 \pi]$ rather than $[-\pi, \pi]$. So let the Fourier frequencies be denoted $\lambda_{s_{j}, N_{j}}=2 \pi s_{j} N_{j}^{-1}$ for $j=1,2$, and define $\bar{\gamma}_{h_{1}, h_{2}}(F)=N^{-2} \sum_{s_{1}=1}^{N_{1}} \sum_{s_{2}=1}^{N_{2}} F\left(\lambda_{s_{1}, N_{1}}, \lambda_{s_{2}, N_{2}}\right) \exp \left\{i h_{1} \lambda_{s_{1}, N_{1}}+i h_{2} \lambda_{s_{2}, N_{2}}\right\}$, which is a discrete approximation to the relation $\gamma_{h_{1}, h_{2}}(F)=\left\langle F Z_{1}^{-h_{1}} Z_{2}^{-h_{2}}\right\rangle$. Fixing $0 \leq h_{1}<N_{1}$, we can consider $\bar{\Sigma}\left(F_{h_{1}}\right)$ to be a $N_{2} \times N_{2}$-dimensional matrix with $j_{2}, k_{2}$ th entry $\bar{\gamma}_{h_{1}, j_{2}-k_{2}}(F)$. The notation suggests that this is an approximation to $\Sigma\left(F_{h_{1}}\right)$ defined at the start of Section 4 of the article. By univariate results [15, p.491] we can write

$$
\bar{\Sigma}\left(F_{h_{1}}\right)=U_{N_{2}}^{*} D_{N_{2}}\left(F_{h_{1}}\right) U_{N_{2}}
$$

with $\left[U_{N_{2}}\right]_{j_{2}, k_{2}}=N^{-1 / 2} \exp \left\{i j_{2} \lambda_{k_{2}, N_{2}}\right\}$ and $D_{N_{2}}\left(F_{h_{1}}\right)$ defined to be a diagonal matrix with $\ell, \ell$ th entry given by $N_{2}^{-1} \sum_{s_{2}=1}^{N_{2}} F\left(\lambda_{\ell, N_{1}}, \lambda_{s_{2}, N_{2}}\right) \exp \left\{i h_{1} \lambda_{s_{2}, N_{2}}\right\}$. The $*$ denotes the complex conjugate transpose.

Next, we construct $\bar{\Sigma}(F)$ as an approximation to $\Sigma(F)$, consisting of Toeplitz blocks $\bar{\Sigma}\left(F_{j_{1}-k_{1}}\right)$ in the $j_{1}, k_{1}$ th slot, with $1 \leq j_{1}, k_{1} \leq N_{1}$. Since the $U_{N_{2}}$ matrices in (B.7) are common to each $\bar{\Sigma}\left(F_{h_{1}}\right)$, we obtain

$$
\bar{\Sigma}(F)=\operatorname{diag}\left(U_{N_{2}}^{*}\right)\left[D_{N_{2}}\left(F_{j_{1}-k_{1}}\right)\right]_{j_{1}, k_{1}=1}^{N_{1}} \operatorname{diag}\left(U_{N_{2}}\right) .
$$

Here $\left[D_{N_{2}}\left(F_{j_{1}-k_{1}}\right)\right]_{j_{1}, k_{1}=1}^{N_{1}}$ denotes a block-Toeplitz matrix consisting of the diagonal matrices $D_{N_{2}}\left(F_{j_{1}-k_{1}}\right)$ in each $j_{1}, k_{1}$ th block, with $1 \leq j_{1}, k_{1} \leq N_{1}$. This block-Toeplitz matrix (with diagonal blocks) can be reformulated as a block-diagonal matrix (with Toeplitz blocks) by application of a permutation matrix $P$. To formalize this, let $P$ be a $N^{2} \times N^{2}$-dimensional permutation matrix that for each $1 \leq k_{1} \leq N_{1}$ takes the $k_{1}$ th row of each block matrix and stacks them in order in the $k_{1}$ th "mega-row", i.e., rows $\left(k_{1}-1\right) N_{2}+1$ through $k_{1} N_{2}$. Then $P\left[D_{N_{2}}\left(F_{j_{1}-k_{1}}\right)\right]_{j_{1}, k_{1}=1}^{N_{1}} P^{\prime}$ is equal to a block-diagonal matrix consisting of Toeplitz matrices $\left[D_{N_{2}}\left(F_{j_{1}-k_{1}}\right)\right]_{\ell \ell}$ in the $\ell$ th block. We denote 
these matrices by $\Sigma\left[F\left(\lambda_{\ell, N_{1}}\right)\right]$, which have $j_{1}, k_{1}$ th entry $N_{1}^{-1} \sum_{s_{1}=1}^{N_{1}} F\left(\lambda_{s_{1}, N_{1}}, \lambda_{\ell, N_{2}}\right) \exp \left\{i\left(j_{1}-\right.\right.$ $\left.\left.k_{1}\right) \lambda_{s_{1}, N_{1}}\right\}$. It then follows that

$$
\bar{F}=\operatorname{diag}\left(U_{N_{2}}^{*}\right) P^{\prime} \operatorname{diag}\left\{\operatorname{diag}\left\{F\left(\lambda_{j_{1}, N_{1}}, \lambda_{\ell_{2}, N_{2}}\right)\right\}_{j_{1}=1}^{N_{1}}\right\}_{\ell_{2}=1}^{N_{2}} P \operatorname{diag}\left(U_{N_{2}}\right) .
$$

Now the middle matrix is a doubly-diagonal matrix consisting of all the values of $F$ at Fourier frequencies, in lexicographical order. Let us abbreviate this matrix by $D_{N}(F)$. Then this approximation $\bar{\Sigma}(F)$ is particularly convenient, since

$$
\bar{\Sigma}(F) \bar{\Sigma}^{-1}(G)=\operatorname{diag}\left(U_{N_{2}}^{*}\right) P^{\prime} D_{N}(F / G) P \operatorname{diag}\left(U_{N_{2}}\right)=\bar{\Sigma}(F / G)
$$

for bounded spectra. At once we find that the trace of this expression is

$$
\sum_{s_{1}=1}^{N_{1}} \sum_{s_{2}=1}^{N_{2}} F\left(\lambda_{s_{1}, N_{1}}, \lambda_{s_{2}, N_{2}}\right) / G\left(\lambda_{s_{1}, N_{1}}, \lambda_{s_{2}, N_{2}}\right)
$$

which when divided by $N^{2}$ will converge to $\langle F / G\rangle$. The extension to multiple $F$ s and $G$ s uses similar arguments. So the proof of the lemma depends on establishing that we can swap $\bar{\Sigma}(F)$ for $\Sigma(F)$.

Consider the base case of the trace of $\Sigma(F) \Sigma^{-1}(G)$, since the same techniques can be generalized to multiple terms. Then

$$
\begin{aligned}
& N^{-2} \operatorname{tr}\left\{\Sigma(F) \Sigma^{-1}(G)\right\}-N^{-2} \operatorname{tr}\left\{\bar{\Sigma}(F) \bar{\Sigma}^{-1}(G)\right\} \\
& =N^{-2} \operatorname{tr}\left\{[\Sigma(F)-\bar{\Sigma}(F)] \Sigma^{-1}(G)\right\}+N^{-2} \operatorname{tr}\left\{\bar{\Sigma}(F) \Sigma^{-1}(G)[\bar{\Sigma}(G)-\Sigma(G)] \bar{\Sigma}^{-1}(G)\right\} .
\end{aligned}
$$

Note that by Lemma A.1 of Dahlhaus and Wefelmeyer [4] $|\operatorname{tr}(A B)| \leq|A||B|$ where $|\cdot|$ denotes the Frobenius norm. Also we use the fact that the Frobenius norm of a product can be broken down in terms of 2-norms $\|\cdot\|_{2}$, so that

$$
\left|\operatorname{tr}\left\{\bar{\Sigma}(F) \Sigma^{-1}(G)[\bar{\Sigma}(G)-\Sigma(G)] \bar{\Sigma}^{-1}(G)\right\}\right| \leq\left\|\bar{\Sigma}(F) \Sigma^{-1}(G)\right\|_{2} \cdot|\bar{\Sigma}(G)-\Sigma(G)| \cdot\left\|\bar{\Sigma}^{-1}(G)\right\|_{2} .
$$

With these results, it will suffice to show the boundedness of

$$
|\Sigma(F)-\bar{\Sigma}(F)|^{2}=\sum_{j_{2}, k_{2}=1}^{N_{2}} \sum_{\left|h_{1}\right|=0}^{N_{1}-1}\left|\gamma_{h_{1}, j_{2}-k_{2}}(F)-\bar{\gamma}_{h_{1}, j_{2}-k_{2}}(F)\right|^{2},
$$

though it is sufficient to consider the absolute sum. The analysis requires a generalization of the Wahba [18] result from time series to spatial random fields. First note that $\bar{\gamma}_{h_{1}, h_{2}}(F)=$ $\sum_{m_{1}, m_{2}} \gamma_{h_{1}+m_{1} N_{1}, h_{2}+m_{2} N_{2}}(F)$ by properties of Fourier frequencies, and thus

$$
\gamma_{h_{1}, j_{2}-k_{2}}(F)-\bar{\gamma}_{h_{1}, j_{2}-k_{2}}(F)=\sum_{m_{1}, m_{2} \neq(0,0)} \gamma_{h_{1}+m_{1} N_{1}, j_{2}-k_{2}+m_{2} N_{2}}(F)
$$


Then the sum of the absolute difference can be bounded as follows:

$$
\begin{aligned}
& \sum_{j_{2}, k_{2}=1}^{N_{2}} \sum_{\left|h_{1}\right|=0}^{N_{1}-1}\left|\gamma_{h_{1}, j_{2}-k_{2}}(F)-\bar{\gamma}_{h_{1}, j_{2}-k_{2}}(F)\right| \\
& \leq \sum_{j_{2}, k_{2}=1}^{N_{2}} \sum_{\left|h_{1}\right|=0}^{N_{1}-1}\left|\sum_{m_{1}, m_{2} \neq(0,0)} \gamma_{h_{1}+m_{1} N_{1}, j_{2}-k_{2}+m_{2} N_{2}}(F)\right| \\
& \leq \sum_{\left|i_{2}\right|<N_{2}}\left(N_{2}-\left|i_{2}\right|\right) \sum_{\left|h_{1}\right|<N_{1}} \sum_{m_{1}, m_{2} \neq(0,0)}\left|\gamma_{h_{1}+m_{1} N_{1}, i_{2}+m_{2} N_{2}}(F)\right| \\
& =\sum_{\left|m_{1}\right|=1}^{\infty} \sum_{m_{2}} \sum_{\left|h_{1}\right|<N_{1}} \sum_{\left|i_{2}\right|<N_{2}}\left(N_{2}-\left|i_{2}\right|\right)\left|\gamma_{h_{1}+m_{1} N_{1}, i_{2}+m_{2} N_{2}}(F)\right| \\
& +\sum_{\left|m_{2}\right|=1}^{\infty} \sum_{\left|h_{1}\right|<N_{1}} \sum_{\left|i_{2}\right|<N_{2}}\left(N_{2}-\left|i_{2}\right|\right)\left|\gamma_{h_{1}, i_{2}+m_{2} N_{2}}(F)\right| \\
& =2 \sum_{\left|m_{1}\right|=2}^{\infty} \sum_{m_{2}} \sum_{\left|h_{1}\right|<N_{1}} \sum_{\left|i_{2}\right|<N_{2}}\left(N_{2}-\left|i_{2}\right|\right)\left|\gamma_{h_{1}+m_{1} N_{1}, i_{2}+m_{2} N_{2}}(F)\right| \\
& +\sum_{m_{2}} \sum_{\left|h_{1}\right|<N_{1}} \sum_{\left|i_{2}\right|<N_{2}}\left(N_{2}-\left|i_{2}\right|\right)\left(\left|\gamma_{h_{1}+N_{1}, i_{2}+m_{2} N_{2}}(F)\right|+\left|\gamma_{h_{1}-N_{1}, i_{2}+m_{2} N_{2}}(F)\right|\right) \\
& +2 \sum_{\left|m_{2}\right|=2}^{\infty} \sum_{\left|h_{1}\right|<N_{1}} \sum_{\left|i_{2}\right|<N_{2}}\left(N_{2}-\left|i_{2}\right|\right)\left|\gamma_{h_{1}, i_{2}+m_{2} N_{2}}(F)\right| \\
& +\sum_{\left|h_{1}\right|<N_{2}} \sum_{\left|i_{2}\right|<N_{2}}\left(N_{2}-\left|i_{2}\right|\right)\left(\left|\gamma_{h_{1}, i_{2}+N_{2}}(F)\right|+\left|\gamma_{h_{1}, i_{2}-N_{2}}(F)\right|\right) \\
& \leq 2 \sum_{\left|m_{1}\right|>N_{1}} \sum_{m_{2}} \sum_{\left|i_{2}\right|<N_{2}}\left(N_{2}-\left|i_{2}\right|\right)\left|\gamma_{m_{1}, i_{2}+m_{2} N_{2}}(F)\right| \\
& +2 \sum_{h_{1}, m_{2}} \sum_{\left|i_{2}\right|<N_{2}}\left(N_{2}-\left|i_{2}\right|\right)\left|\gamma_{h_{1}, i_{2}+m_{2} N_{2}}(F)\right| \\
& +2 N_{2} \sum_{\left|m_{2}\right|>N_{2}} \sum_{\left|h_{1}\right|<N_{1}}\left|\gamma_{h_{1}, m_{2}}(F)\right|+2 \sum_{h_{1}} \sum_{\left|i_{2}\right|<N_{2}}\left|i_{2}\right|\left|\gamma_{h_{1}, i_{2}}(F)\right| \\
& \leq 2 C \sum_{\left|m_{1}\right|>N_{1}} \sum_{\left|i_{2}\right|<N_{2}}\left|i_{2}\right|\left|\gamma_{m_{1}, i_{2}}(F)\right|+2 N_{2} \sum_{\left|m_{1}\right|>N_{1}} \sum_{\left|m_{2}\right|>N_{2}}\left|\gamma_{m_{1}, m_{2}}(F)\right| \\
& +2 C \sum_{h_{1}} \sum_{\left|i_{2}\right|<N_{2}}\left|i_{2}\right|\left|\gamma_{h_{1}, i_{2}}(F)\right|+2 N_{2} \sum_{h_{1}} \sum_{\left|m_{2}\right|>N_{2}}\left|\gamma_{h_{1}, m_{2}}(F)\right| \\
& +2 N_{2} \sum_{\left|m_{2}\right|>N_{2}} \sum_{\left|h_{1}\right|>N_{1}}\left|\gamma_{h_{1}, m_{2}}(F)\right|+2 \sum_{h_{1}} \sum_{\left|i_{2}\right|<N_{2}}\left|i_{2}\right|\left|\gamma_{h_{1}, i_{2}}(F)\right| \text {, }
\end{aligned}
$$

where $C>0$ denotes a generic constant. At this point we utilize our definition of $\mathcal{F}$ to conclude that the entire sum is bounded as $N_{*} \rightarrow \infty$. As a result, the trace approximation has error of order $N^{-2}$ (or $N_{*}^{-2}$ ), which is also the approximation error in passing to the Riemann integral.

Proof of Lemma 4.2. We first consider the biased acf estimates, and determine the bias of the corresponding spectral estimate. Recall that $I_{\widetilde{\beta}}$ is the periodogram of the random field $\mathbb{W}^{N}$, 
and its expectation is

$$
\begin{aligned}
\mathbb{E} I_{\widetilde{\beta}}\left(\lambda_{1}, \lambda_{2}\right) & =\sum_{s_{1}, t_{1}=1}^{N_{1}} \sum_{s_{2}, t_{2}=1}^{N_{2}} \mathbb{E} \gamma_{t_{1}-s_{1}, t_{2}-s_{2}}(I) \exp \left\{-i\left[\lambda_{1}\left(t_{1}-s_{1}\right)+\lambda_{2}\left(t_{2}-s_{2}\right)\right]\right\} \\
& =\sum_{\left|h_{1}\right|<N_{1}} \sum_{\left|h_{2}\right|<N_{2}} \gamma_{h_{1}, h_{2}}(\widetilde{F}) \exp \left\{-i\left[\lambda_{1} h_{1}+\lambda_{2} h_{2}\right]\right\}\left(1-\frac{\left|h_{1}\right|}{N_{1}}\right)\left(1-\frac{\left|h_{2}\right|}{N_{2}}\right) .
\end{aligned}
$$

Then its bias, as an estimator of $\widetilde{F}\left(\lambda_{1}, \lambda_{2}\right)$, equals

$$
\begin{aligned}
& \sum_{\left|h_{1}\right| \geq N_{1}} \sum_{\left|h_{2}\right| \geq N_{2}} \gamma_{h_{1}, h_{2}}(\widetilde{F}) \exp \left\{-i\left[\lambda_{1} h_{1}+\lambda_{2} h_{2}\right]\right\} \\
& +\sum_{\left|h_{1}\right|<N_{1}} \sum_{\left|h_{2}\right|<N_{2}} \gamma_{h_{1}, h_{2}}(\widetilde{F}) \exp \left\{-i\left[\lambda_{1} h_{1}+\lambda_{2} h_{2}\right]\right\} \cdot\left(-\frac{\left|h_{1}\right|}{N_{1}}-\frac{\left|h_{2}\right|}{N_{2}}+\frac{\left|h_{1}\right|\left|h_{2}\right|}{N_{1} N_{2}}\right) .
\end{aligned}
$$

In contrast to $I_{\widetilde{\beta}}$, the bias of $\widehat{I}_{\widetilde{\beta}}$ involves only the first term of (B.8). Using the conditions implied by the set $\mathcal{F}$, this term (B.8) is $O\left(N_{*}^{-2}\right)$, which means that $\widetilde{F}$ can be swapped for $\mathbb{E} \widehat{I}_{\widetilde{\beta}}$ in the asymptotic calculations without cost. But for $I_{\widetilde{\beta}}$, the bias contains additional terms $B_{1}\left(\lambda_{1}, \lambda_{2}\right) N_{1}^{-1}$ and $B_{2}\left(\lambda_{1}, \lambda_{2}\right) N_{2}^{-1}$, up to terms that are $O\left(N^{-2}\right)$. In particular, the bias of $I_{\widetilde{\beta}}$ is $O\left(N_{*}^{-1}\right)$, which differs from the time series case; cf. results in Guyon [5]. Without the extra bias correction furnished by $B_{1} / N_{1}+B_{2} / N_{2}$, the asymptotic bias dominates ${ }^{1}$, although the estimator is still consistent.

Once the bias in the estimates is accounted for, such that the error is at most $O\left(N^{-2}\right)$, then multiplication of the estimator minus $\widetilde{F}$ by $N$ produces error of $O\left(N^{-1}\right)$, so that we can proceed by analyzing the mean-centered periodogram estimator. Further results, which are needed to prove the Lemma's claims, involve generalizations of Theorems 4.3.1 and 4.3.2 of Brillinger [1] to the spatial field case. To establish joint convergence, we use the Cramer-Wold device and linearity of the forms; so without loss of generality consider the problem with a single weighting function $G$.

Consider $N^{-1} \sum_{\lambda} G(\lambda)\left[\widehat{I}_{\widetilde{\beta}}(\lambda)-\widetilde{F}(\lambda)\right]$, where the sum is a double sum over pairs of Fourier frequencies, noting that we can swap the mean $\mathbb{E} \widehat{I}_{\widetilde{\beta}}(\lambda)$ for $\widetilde{F}$. (The same arguments will also apply to $I_{\widetilde{\beta}}-B_{1} / N_{1}-B_{2} / N_{2}$.) The variance of $N^{-1} \sum_{\lambda} G(\lambda) \widehat{I}_{\widetilde{\beta}}(\lambda)$ is given by an expansion involving cumulants. Let $\vartheta_{\ell m}$ and $\varphi_{\ell m}$ for $\ell, m=1,2$ be defined as various signed combinations of frequencies $\lambda_{1}, \lambda_{2}, \omega_{1}, \omega_{2}$, given as follows:

$$
\left[\begin{array}{ll}
\left(-\lambda_{1},-\lambda_{2}\right) & \left(\lambda_{1}, \lambda_{2}\right) \\
\left(-\omega_{1},-\omega_{2}\right) & \left(\omega_{1}, \omega_{2}\right)
\end{array}\right]=\left[\begin{array}{ll}
\left(\vartheta_{11}, \varphi_{11}\right) & \left(\vartheta_{12}, \varphi_{12}\right) \\
\left(\vartheta_{21}, \varphi_{21}\right) & \left(\vartheta_{22}, \varphi_{22}\right)
\end{array}\right]
$$

For convenience in the calculations, we utilize $I_{\widetilde{\beta}}$ minus its mean, instead of $\widehat{I}_{\widetilde{\beta}}$. The variance is then

$$
\begin{aligned}
& N^{-2} \sum_{\lambda, \omega} G(\lambda) G(\omega) \operatorname{cum}\left(I_{\widetilde{\beta}}(\lambda), I_{\widetilde{\beta}}(\omega)\right) \\
& =N^{-6} \sum_{\nu, \lambda, \omega} G(\lambda) G(\omega) \operatorname{cum}\left(\widetilde{\mathbb{W}}\left(\vartheta_{\ell m}, \varphi_{\ell m}\right):(\ell, m) \in \nu_{1}, \ldots, \widetilde{\mathbb{W}}\left(\vartheta_{\ell m}, \varphi_{\ell m}\right):(\ell, m) \in \nu_{q}\right),
\end{aligned}
$$

\footnotetext{
${ }^{1}$ This effect gets worse with increasing dimension of the field, as is seen by computing $\Pi_{j=1}^{d}\left(1-\left|h_{j}\right| N_{j}^{-1}\right)$, which equals $1+O\left(N_{*}^{-1}\right)$ with $N_{*}=\min \left\{N_{1}, N_{2}, \ldots, N_{d}\right\}$.
} 
where the sum is over all indecomposable partitions $\nu$ of the table

$$
\left[\begin{array}{ll}
(1,1) & (1,2) \\
(2,1) & (2,2)
\end{array}\right]
$$

Therefore the only proper partitions that need be considered are $\{(1,1),(2,1)\} \bigcup\{(1,2),(2,2)\}$ and $\{(1,1),(2,2)\} \bigcup\{(1,2),(2,1)\}$. We proceed to calculate the relevant asymptotic cumulants:

$$
\begin{aligned}
& \operatorname{cum}\left(\widetilde{\mathbb{W}}\left(-\lambda_{1},-\lambda_{2}\right), \widetilde{\mathbb{W}}\left(-\omega_{1},-\omega_{2}\right)\right) \\
& =\sum_{t, s} \gamma_{s_{1}-t_{1}, s_{2}-t_{2}}(\widetilde{F}) \exp \left\{i\left(\lambda_{1} t_{1}+\lambda_{2} t_{2}+\omega_{1} s_{1}+\omega_{2} s_{2}\right)\right\} \\
& =\sum_{h_{1}, h_{2}} \gamma_{h_{1}, h_{2}}(\widetilde{F}) \exp \left\{i \omega_{1} h_{1}+i \omega_{2} h_{2}\right\}\left(\sum_{t_{1}=1}^{N_{1}-h_{1}} \sum_{t_{2}=1}^{N_{2}-h_{2}} \exp \left\{i\left(\lambda_{1}+\omega_{1}\right) t_{1}+i\left(\lambda_{2}+\omega_{2}\right) t_{2}\right\}\right),
\end{aligned}
$$

which is order $N^{2}$ when $\lambda_{1}=-\omega_{1}$ and $\lambda_{2}=-\omega_{2}$, but is lower order otherwise. In the former case, the cumulant is asymptotic to $N^{2} \widetilde{F}\left(-\omega_{1},-\omega_{2}\right)$. The next cumulant is that for $\widetilde{\mathbb{V}}\left(\lambda_{1}, \lambda_{2}\right)$ times $\widetilde{\mathbb{W}}\left(\omega_{1}, \omega_{2}\right)$, but the analysis is just like the previous case (only with minus signs deleted), and the asymptotic in the case $\lambda_{1}=-\omega_{1}$ and $\lambda_{2}=-\omega_{2}$ is $N^{2} \widetilde{F}\left(\omega_{1}, \omega_{2}\right)$. Next, we have

$$
\begin{aligned}
& \operatorname{cum}\left(\widetilde{\mathbb{W}}\left(-\lambda_{1},-\lambda_{2}\right), \widetilde{\mathbb{W}}\left(\omega_{1}, \omega_{2}\right)\right) \\
& =\sum_{t, s} \gamma_{s_{1}-t_{1}, s_{2}-t_{2}}(\widetilde{F}) \exp \left\{i\left(\lambda_{1} t_{1}+\lambda_{2} t_{2}-\omega_{1} s_{1}-\omega_{2} s_{2}\right)\right\} \\
& =\sum_{h_{1}, h_{2}} \gamma_{h_{1}, h_{2}}(\widetilde{F}) \exp \left\{-i \omega_{1} h_{1}-i \omega_{2} h_{2}\right\}\left(\sum_{t_{1}=1}^{N_{1}-h_{1}} \sum_{t_{2}=1}^{N_{2}-h_{2}} \exp \left\{i\left(\lambda_{1}-\omega_{1}\right) t_{1}+i\left(\lambda_{2}-\omega_{2}\right) t_{2}\right\}\right),
\end{aligned}
$$

which is order $N^{2}$ when $\lambda_{1}=\omega_{1}$ and $\lambda_{2}=\omega_{2}$, but is lower order otherwise, and thus is asymptotic to $N^{2} \widetilde{F}\left(\omega_{1}, \omega_{2}\right)$. Finally, the cumulant of $\widetilde{\mathbb{W}}\left(\lambda_{1}, \lambda_{2}\right)$ and $\widetilde{\mathbb{W}}\left(-\omega_{1},-\omega_{2}\right)$ is similarly computed, with the result of $N^{2} \widetilde{F}\left(-\omega_{1},-\omega_{2}\right)$.

There is also the cumulant involving all four discrete Fourier Transforms, namely

$$
\begin{aligned}
& \operatorname{cum}\left(\widetilde{\mathbb{W}}\left(-\lambda_{1},-\lambda_{2}\right), \widetilde{\mathbb{W}}\left(\lambda_{1}, \lambda_{2}\right), \widetilde{\mathbb{W}}\left(-\omega_{1},-\omega_{2}\right), \widetilde{\mathbb{W}}\left(\omega_{1}, \omega_{2}\right)\right) \\
& =\sum_{t, s, m, n} \operatorname{cum}\left(W_{t}, W_{s}, W_{m}, W_{n}\right) e^{\left\{i\left(\lambda_{1} t_{1}+\lambda_{2} t_{2}-\lambda_{1} s_{1}-\lambda_{2} s_{2}+\omega_{1} m_{1}+\omega_{2} m_{2}-\omega_{1} n_{1}-\omega_{2} n_{2}\right)\right\}} \\
& \sim N^{2} \sum_{h_{1}, h_{2}, k_{1}, k_{2}, \ell_{1}, \ell_{2}} \gamma_{h_{1}, h_{2}, k_{1}, k_{2}, \ell_{1}, \ell_{2}} \exp \left\{i\left(-\lambda_{1} h_{1}-\lambda_{2} h_{2}+\omega_{1} k_{1}+\omega_{2} k_{2}-\omega_{1} \ell_{1}-\omega_{2} \ell_{2}\right)\right\} \\
& =N^{2} \widetilde{F F}\left(\lambda_{1}, \lambda_{2},-\omega_{1},-\omega_{2}, \omega_{1}, \omega_{2}\right) .
\end{aligned}
$$

Then consolidating, the variance of $N^{-1} \sum_{\lambda} G(\lambda) I_{\widetilde{\beta}}(\lambda)$ is asymptotic to

$$
\begin{aligned}
& N^{-4} \sum_{\lambda} \sum_{\omega} G(\lambda) G(\omega) \widetilde{F F}\left(\lambda_{1}, \lambda_{2},-\omega_{1},-\omega_{2}, \omega_{1}, \omega_{2}\right) \\
& +N^{-2} \sum_{\lambda}\left(G(\lambda) G(-\lambda)+G^{2}(\lambda)\right) \widetilde{F}^{2}(\lambda) \\
& \sim\langle\langle G(\lambda) G(\omega) \widetilde{F F}(\lambda,-\omega, \omega)\rangle\rangle+\left\langle\left(G(\lambda) G(-\lambda)+G^{2}(\lambda)\right) \widetilde{F}^{2}(\lambda)\right\rangle .
\end{aligned}
$$


This is the main calculation, but some details remain. The above expression provides the asymptotic variance, and once generalized to a joint result over several functions $G_{j}$ yields the stated matrix $V$. Our results have been derived for $N^{-1}\left\langle G_{j}\left(\widehat{I}_{\widetilde{\beta}}-\widetilde{F}\right)\right\rangle_{N}$; for $N^{-1}\left\langle G_{j}\left(\widehat{I}_{\widetilde{\beta}}-\widetilde{F}\right)\right\rangle$ we can proceed as in Theorem 5.10.2 of Brillinger (2001), using the boundedness of the functions $G_{j}$ and the above DFT results to bound the difference of $I_{\widetilde{\beta}}\left(2 \pi t_{1} N_{1}^{-1}, 2 \pi t_{2} N_{2}^{-1}\right)$ and $I_{\widetilde{\beta}}(\lambda)$ over the region $\left\{\lambda \in\left[2 \pi t_{1} N_{1}^{-1}, 2 \pi\left(t_{1}+1\right) N_{1}^{-1}\right] \times\left[2 \pi t_{2} N_{2}^{-1}, 2 \pi\left(t_{2}+1\right) N_{2}^{-1}\right]\right\}$ by $O_{P}\left(N^{-1}\right)$.

Finally, higher order moments of $N^{-1}\left\langle G\left(\widehat{I}_{\widetilde{\beta}}-\widetilde{F}\right)\right\rangle$ will be asymptotically negligible due to the higher order $\left(k>3\right.$ in Condition $\left.B_{k}\right)$ cumulant conditions, which is proved along the lines of the combinatorial analysis of Theorem 1 in McElroy and Holan [11]. This asymptotic structure for the cumulants indicates a limiting normal distribution, as stated.

Proof of Theorem 4.1. First note that $N_{*} \rightarrow \infty$ implies that $N \rightarrow \infty$. We begin with the analysis of $\widehat{\beta}$. From (3.2) and (3.4) in the article, we obtain

$$
\begin{aligned}
\widehat{\beta}_{Q M L E}-\widetilde{\beta} & =\left[\widetilde{X}^{\prime} \Sigma\left(F_{\widehat{\theta}_{Q M L E}}^{-1}\right) \widetilde{X}\right]^{-1} \widetilde{X}^{\prime} \Sigma\left(F_{\widehat{\theta}_{Q M L E}^{-1}}^{-}\right)(Y-\widetilde{X} \widetilde{\beta}) \\
\widehat{\beta}_{M L E}-\widetilde{\beta} & =\left[\tilde{X}^{\prime} \Sigma^{-1}\left(F_{\widehat{\theta}_{M L E}}\right) \widetilde{X}\right]^{-1} \widetilde{X}^{\prime} \Sigma^{-1}\left(F_{\widehat{\theta}_{M L E}}\right)(Y-\widetilde{X} \widetilde{\beta}) .
\end{aligned}
$$

So the regression errors in both cases are linear functions of $W$; consistency follows from $\mathbf{A 2}$ and the condition that $\left(\tilde{X}^{\prime} \widetilde{X}\right)^{-1}$ tends to the zero matrix. By the cumulant conditions that we assume (A3), the random field $\mathbb{W}^{N}$ satisfies a central limit theorem. Moreover, we can replace the estimates $\hat{\theta}$ by their limits $\widetilde{\theta}$ (their consistency is proved below) because the error in doing so is of lesser order by Lemma 4.1. Moreover by the same Lemma, $N^{-1} \widetilde{X}^{\prime} \Sigma^{-1}\left(F_{\widetilde{\theta}}\right) W$ has the same asymptotics as $N^{-1} \widetilde{X}^{\prime} \Sigma\left(F_{\widetilde{\theta}}^{-1}\right) W$, which is asymptotically normal with mean zero and covariance

$$
N^{-2} \widetilde{X}^{\prime} \Sigma\left(F_{\widetilde{\theta}}^{-1}\right) \Sigma(\widetilde{F}) \Sigma\left(F_{\widetilde{\theta}}^{-1}\right) \widetilde{X}
$$

This result follows from the argument used in Theorem 2 of Mardia and Marshall [10], together with $\mathbf{A 2}$ and the assumption that $\left(\widetilde{X}^{\prime} \tilde{X}\right)^{-1}$ tends to a zero matrix as $N_{*} \rightarrow \infty$ (plus the central limit theorem for $\mathbb{W}^{N}$ by $\mathbf{A 3}$, as our data process may be non-Gaussian). Now applying the matrix $M_{X}(\widetilde{\theta})$ to the asymptotic normality result yields the limit theorems for the regression QMLE and MLE. This argument holds for the exact Whittle estimates, but as in the proof of Lemma 4.2, can be extended to the case of approximate Whittle estimates as well (see next paragraph). Below, we will show that the MLE and QMLE for $\theta$ can be expressed as a quadratic form in the data (asymptotically), and hence is uncorrelated with the regression estimates when the third cumulants of the data process are zero.

Now we consider the asymptotics for the parameter $\theta$. First we examine the exact and approximate QMLEs. One may develop an error expression along the lines given for Lemma 3.1.1 of Taniguchi and Kakizawa [15], with $\widehat{\theta}$ denoting the exact QMLE and $\widehat{\theta}_{N}$ the approximate QMLE:

$$
\begin{aligned}
(\widehat{\theta}-\widetilde{\theta}) & =o_{P}(1)-\left[\nabla \nabla^{\prime} \mathrm{KL}\left(F_{\widetilde{\theta}}, \widetilde{F}\right)\right]^{-1}\left\langle\nabla F_{\widetilde{\theta}}^{-1}\left(\widehat{I}_{\widetilde{\beta}}-\widetilde{F}\right)\right\rangle \\
\left(\widehat{\theta}_{N}-\widetilde{\theta}\right) & =o_{P}(1)-\left[\nabla \nabla^{\prime} \mathrm{KL}_{N}\left(F_{\widetilde{\theta}}, \widetilde{F}\right)\right]^{-1}\left\langle\nabla F_{\widetilde{\theta}}^{-1}\left(\widehat{I}_{\widetilde{\beta}}-\widetilde{F}\right)\right\rangle_{N},
\end{aligned}
$$

which are developed by Taylor Series expansion in each case. In order to write down this expansion, we must replace $\widehat{I}_{\widehat{\beta}}$ by $\widehat{I}_{\widetilde{\beta}}$, which uses the consistency of $\widehat{\beta}$. Now in the proof of Lemma 4.2 it is 
established that $\mathrm{KL}$ and $\mathrm{KL}_{N}$ differ in their stochastic terms of order $O_{P}\left(N^{-1}\right)$, whereas the $\log$ determinant terms differ by $O\left(N^{-2}\right)$ via the definition of the Riemann integral. These types of results also hold for the difference of the gradients of the KL functions, since $\nabla F_{\theta}^{-1}$ is continuous by assumption. Then we apply Lemma 4.2 to the above convergences, as $\nabla F_{\theta}^{-1}=-\nabla F_{\theta} \cdot F_{\theta}^{-2}$, which is continuous by assumption A2. Noting the mirror symmetry of $F$, we see that $V(\theta)$ has the form stated in the theorem.

Next consider the MLE case, where the scaled log Gaussian likelihood is $\mathcal{L}$ given in (3.3) of the article. The method of proof follows the treatment in Section 4.1 of Taniguchi and Kakizawa [15], but with their Lemma 4.1.2 replaced by our Lemma 4.1. Also note that many of the calculations are identical with those of Mardia and Marshall [10], though our regularity conditions together with Lemma 4.1 essentially verify condition (iii) of their Theorem 2 (also those authors assume a Gaussian spatial process, whereas we allow small departures from normality). First

$$
0=\nabla \log \mathcal{L}\left(\widehat{\theta}_{M L E}\right)=\nabla \log \mathcal{L}(\widetilde{\theta})+\nabla \nabla^{\prime} \log \mathcal{L}(\widetilde{\theta})(\widehat{\theta}-\widetilde{\theta})+\frac{1}{2} J\left(\theta^{*}, \widehat{\theta}-\widetilde{\theta}\right)
$$

for some $\theta^{*}$ such that $\left\|\theta^{*}-\widetilde{\theta}\right\| \leq\|\widehat{\theta}-\theta\|$, with $\|\cdot\|$ the Euclidean norm. Here $J$ is a 3-tensor that linearly depends on $\widehat{\theta}-\widetilde{\theta}$; if $\widehat{\theta}-\widetilde{\theta}=O_{P}\left(N^{-1}\right)$, then this term will be $O_{P}\left(N^{-3}\right)$. As in Taniguchi and Kakizawa [15, p.180] set $U_{N}=N(\widehat{\theta}-\widetilde{\theta})$ and $Z_{N}(\theta)=N^{-1} \nabla \log L(\theta)$, which equals $X^{\prime} \Sigma^{-1}\left(F_{\theta}\right) \nabla \Sigma\left(F_{\theta}\right) \Sigma^{-1}\left(F_{\theta}\right) X /(2 N)-\operatorname{tr}\left(\Sigma^{-1}\left(F_{\theta}\right) \nabla \Sigma\left(F_{\theta}\right)\right) /(2 N)$. Here $\nabla \Sigma\left(F_{\theta}\right)$ is a shorthand for an array of matrices, each of which is the derivative of $\Sigma\left(F_{\theta}\right)$ with respect to one of the $\theta_{j}$. Then from (B.9) we have $U_{N}=-\left[N^{-2} H_{N}(\widetilde{\theta})\right]^{-1} Z_{N}(\widetilde{\theta})$ plus terms of order $N^{-2}$, with $H_{N}$ equal to the Hessian of $\mathcal{L}$. The mean of $Z_{N}(\theta)$ is given by

$$
\frac{1}{2 N} \operatorname{tr}\left\{\Sigma^{-1}\left(F_{\theta}\right) \nabla \Sigma\left(F_{\theta}\right) \Sigma^{-1}\left(F_{\theta}\right)\left[\Sigma(\widetilde{F})-\Sigma\left(F_{\theta}\right)\right]\right\}=O\left(N^{-1}\right)+\frac{N}{2}\left\langle\nabla F_{\theta} F_{\theta}^{-2}\left[\widetilde{F}-F_{\theta}\right]\right\rangle,
$$

where the second equality follows from Lemma 4.1. The final integral is actually equal to the gradient of $\operatorname{KL}\left(F_{\theta}, \widetilde{F}\right)$, and thus is identically zero when $\theta$ equals the pseudo-true value, no matter whether the model is correctly specified or not. If the fourth cumulants are zero, then

$$
\operatorname{Var}\left[Z_{N}(\theta)\right]=\frac{1}{2 N^{2}} \operatorname{tr}\left(\Sigma^{-1}\left(F_{\theta}\right) \nabla \Sigma\left(F_{\theta}\right) \Sigma^{-1}\left(F_{\theta}\right) \Sigma(\widetilde{F}) \Sigma^{-1}\left(F_{\theta}\right) \nabla^{\prime} \Sigma\left(F_{\theta}\right) \Sigma^{-1}\left(F_{\theta}\right) \Sigma(\widetilde{F})\right),
$$

which by Lemma 4.1 converges to the matrix $V(\theta) / 4$. The more general expression for the variance - in the case of nonzero fourth cumulants - follows the same lines as the proof of Lemma 4.1, and by that result converges to the more general expression of $V(\theta) / 4$. Higher order cumulants of $Z_{N}$ are analyzed along the lines offered in Taniguchi and Kakizawa [15], only replacing their use of Lemma 4.1.2 (e.g., in their equation (4.1.24)) by our Lemma 4.1. Hence $Z_{N}(\theta) \stackrel{\mathcal{L}}{\Longrightarrow} \mathcal{N}(0, V(\theta) / 4)$. Also we have

$$
\begin{aligned}
2 \partial_{j} \partial_{k} \log \mathcal{L}(\theta) & =-X^{\prime} \Sigma^{-1}\left(F_{\theta}\right) \partial_{j} \Sigma\left(F_{\theta}\right) \Sigma^{-1}\left(F_{\theta}\right) \partial_{k} \Sigma\left(F_{\theta}\right) \Sigma^{-1}\left(F_{\theta}\right) X \\
& -X^{\prime} \Sigma^{-1}\left(F_{\theta}\right) \partial_{k} \Sigma\left(F_{\theta}\right) \Sigma^{-1}\left(F_{\theta}\right) \partial_{j} \Sigma\left(F_{\theta}\right) \Sigma^{-1}\left(F_{\theta}\right) X \\
& +X^{\prime} \Sigma^{-1}\left(F_{\theta}\right) \partial_{j} \partial_{k} \Sigma\left(F_{\theta}\right) \Sigma^{-1}\left(F_{\theta}\right) X \\
& -\operatorname{tr}\left\{\Sigma^{-1}\left(F_{\theta}\right) \partial_{j} \partial_{k} \Sigma\left(F_{\theta}\right)-\Sigma^{-1}\left(F_{\theta}\right) \partial_{j} \Sigma\left(F_{\theta}\right) \Sigma^{-1}\left(F_{\theta}\right) \partial_{k} \Sigma\left(F_{\theta}\right)\right\},
\end{aligned}
$$

which is twice the Hessian matrix $H_{N}(\theta)$. The expectation is

$$
\begin{aligned}
\mathbb{E} H_{N}(\theta) & =-\operatorname{tr}\left\{\Sigma^{-1}\left(F_{\theta}\right) \nabla \Sigma\left(F_{\theta}\right) \Sigma^{-1}\left(F_{\theta}\right) \nabla^{\prime} \Sigma\left(F_{\theta}\right) \sigma^{-1}\left(F_{\theta}\right) \Sigma(\widetilde{F})\right\} \\
& +\operatorname{tr}\left\{\Sigma^{-1}\left(F_{\theta}\right) \nabla \nabla^{\prime} \Sigma\left(F_{\theta}\right) \Sigma^{-1}\left(F_{\theta}\right) \Sigma(\widetilde{F})\right\} / 2 \\
& -\operatorname{tr}\left\{\Sigma^{-1}\left(F_{\theta}\right) \nabla \nabla^{\prime} \Sigma\left(F_{\theta}\right)-\Sigma^{-1}\left(F_{\theta}\right) \nabla \Sigma\left(F_{\theta}\right) \Sigma^{-1}\left(F_{\theta}\right) \nabla^{\prime} \Sigma\left(F_{\theta}\right)\right\} / 2,
\end{aligned}
$$


and applying Lemma 4.1 we have $N^{-2} \mathbb{E} H_{N}(\theta)$ tends to

$$
-\left\langle F_{\theta}^{-3} \nabla F_{\theta} \nabla^{\prime} F_{\theta} \widetilde{F}\right\rangle+\left\langle F_{\theta}^{-2} \nabla \nabla^{\prime} F_{\theta} \widetilde{F}\right\rangle / 2-\left\langle F_{\theta}^{-1} \nabla \nabla^{\prime} F_{\theta}\right\rangle / 2+\left\langle F_{\theta}^{-2} \nabla F_{\theta} \nabla^{\prime} F_{\theta}\right\rangle / 2,
$$

which is half the Hessian of $\operatorname{KL}\left(F_{\theta}, \widetilde{F}\right)$, i.e., $H(\theta) / 2$. Also by Lemma 4.1 we obtain

$$
\operatorname{Var}\left(N^{-2} \nabla \nabla^{\prime} \log L(\theta)\right) \sim \frac{1}{2 N^{2}}\left\langle\left(2 \nabla F_{\theta} \nabla^{\prime} F_{\theta} \widetilde{F}^{-2}-\nabla \nabla^{\prime} F_{\theta} \widetilde{F}^{-1}\right)^{2}\right\rangle
$$

as $N_{*} \rightarrow \infty$, and hence $N^{-2} H_{N}(\widetilde{\theta}) \stackrel{P}{\longrightarrow} H(\widetilde{\theta}) / 2$. Then $H(\widetilde{\theta}) U_{N} \stackrel{\mathcal{L}}{\longrightarrow} \mathcal{N}(0, V(\widetilde{\theta}))$, and the stated result follows.

Proof of Theorem 4.2. First note that $N_{*} \rightarrow \infty$ implies that $N \rightarrow \infty$. All integrals are multidimensional. Let $\|\cdot\|$ denote the Euclidean norm. Let $p(\theta \mid X)$ denote the posterior for $\phi=\left[\theta^{\prime}, \beta^{\prime}\right]^{\prime}$, which is proportional to the Gaussian likelihood $p(X \mid \phi)$ times $p(\phi)$, the prior for the parameter vector. The posterior estimate is the vector quantity $\widehat{\phi}=\int \phi p(\phi \mid X) d \phi$; we seek to show that it converges in probability to $\widetilde{\phi}$, the true parameter value. We further develop the likelihood as

$$
\begin{aligned}
p(X \mid \phi) & =\exp \left[-\frac{N^{2}}{2}\left\{\log (2 \pi)+\mathcal{L}_{N}(\theta, \beta)\right\}\right] \\
\mathcal{L}_{N}(\theta, \beta) & =N^{-2} \log \left|\Sigma\left(F_{\theta}\right)\right|+N^{-2}(Y-\widetilde{X} \beta)^{\prime} \Sigma^{-1}\left(F_{\theta}\right)(Y-\widetilde{X} \beta) .
\end{aligned}
$$

We next expand the quadratic form so as to relate it to the true $\widetilde{\beta}$ parameter:

$$
\begin{aligned}
(Y-\widetilde{X} \beta)^{\prime} \Sigma^{-1}\left(F_{\theta}\right)(Y-\widetilde{X} \beta) & =(Y-\widetilde{X} \widetilde{\beta})^{\prime} \Sigma^{-1}\left(F_{\theta}\right)(Y-\widetilde{X} \widetilde{\beta}) \\
& +2(\widetilde{\beta}-\beta)^{\prime} \widetilde{X}^{\prime} \Sigma^{-1}\left(F_{\theta}\right)(Y-\widetilde{X} \widetilde{\beta}) \\
& +(\widetilde{\beta}-\beta)^{\prime} \widetilde{X}^{\prime} \Sigma^{-1}\left(F_{\theta}\right) \widetilde{X}(\widetilde{\beta}-\beta)
\end{aligned}
$$

The first term on the right hand represents the same quadratic form, but with $\widetilde{\beta}$ replacing $\beta$; therefore it is a quadratic form in $W$. The second term is linear in $W$, so as in the proof of Theorem 4.1 will obey a central limit theorem and is $O_{P}(N)$. The final term is deterministic, and under the conditions of the theorem is of order $N^{2}$. As a result,

$$
\mathcal{L}_{N}(\theta, \beta)=\mathcal{L}_{N}(\theta, \widetilde{\beta})+E_{N}+Q(\theta, \beta)
$$

where $E_{N}$ is an error term tending to zero in probability and $Q(\theta, \beta)=(\widetilde{\beta}-\beta)^{\prime} M(\theta)(\widetilde{\beta}-\beta)$. Note that by our assumptions on $M(\theta)$, the quadratic form $Q(\theta, \beta)$ is positive whenever $\beta \neq \widetilde{\beta}$, for any $\theta$, and moreover is bounded above by $\bar{\Lambda}\|\widetilde{\beta}-\beta\|^{2}$ for all $\theta$, where $\bar{\Lambda}$ bounds all the eigenvalues of $M(\theta)$ for all $\theta$. Also $\underline{\Lambda}$ is the lower (positive) bound on the eigenvalues of $M(\theta)$.

So for any $\epsilon>0$, define neighborhoods $N_{\epsilon}(\widetilde{\theta})=\{\theta:\|\theta-\widetilde{\theta}\|<\epsilon\}$ and similarly $N_{\epsilon}(\widetilde{\beta})$. We can then decompose the parameter space (because all parameters are contained in a compact subset of Euclidean space) into $N_{\epsilon}(\widetilde{\theta}) \times N_{\epsilon}(\widetilde{\beta})$ and its complement. This complement can be broken into two overlapping regions, $R_{1}$ and $R_{2}$, where $R_{1}=N_{\epsilon}^{c}(\widetilde{\theta})$ and $R_{2}=N_{\epsilon}^{c}(\widetilde{\beta})$. The complements are taken in the entire product parameter space, so $R_{1}$ corresponds to $\|\widetilde{\theta}-\theta\|>\epsilon$ but $\beta$ is unconstrained, while $R_{2}$ corresponds to $\|\widetilde{\beta}-\beta\|>\epsilon$ but $\theta$ is unconstrained. The intersection region $R_{1} \cap R_{2}$ is characterized by both constraints being true. In order to get disjoint regions, let $\bar{R}_{1}=R_{1} \backslash\left(R_{1} \bigcap R_{2}\right)$ 
and $\bar{R}_{2}=R_{2} \backslash\left(R_{1} \bigcap R_{2}\right)$. Then we decompose the vector parameter error as

$$
\begin{aligned}
\widehat{\phi}-\widetilde{\phi} & =\frac{\int_{N_{\epsilon}(\widetilde{\theta}) \times N_{\epsilon}(\widetilde{\beta})}(\phi-\widetilde{\phi}) \exp \left\{-\frac{N^{2}}{2} \mathcal{L}_{N}(\theta, \beta)\right\} p(\phi) d \phi}{\int \exp \left\{-\frac{N^{2}}{2} \mathcal{L}_{N}(\theta, \beta)\right\} p(\phi) d \phi} \\
& +\frac{\int \bar{R}_{1} \cup \bar{R}_{2} \bigcup\left(R_{1} \cap R_{2}\right)}{\int \exp \{-\widetilde{\phi}) \exp \left\{-\frac{N^{2}}{2} \mathcal{L}_{N}(\theta, \beta)\right\} p(\phi) d \phi} .
\end{aligned}
$$

The integrands $\phi-\widetilde{\phi}$ are vectors, and the integrals are interpreted as integrating each component and stacking the result. The first summand has Euclidean norm bounded by $\epsilon$ with probability one. Focusing on the second term, we proceed to develop two key bounds [see 9, 7, for a similar approach]:

$$
\mathcal{L}_{N}(\theta, \widetilde{\beta})-\mathcal{L}_{N}(\widetilde{\theta}, \widetilde{\beta}) \geq \bar{C}(\theta)
$$

for all $\theta \in N_{\epsilon}^{c}(\widetilde{\theta})$ with probability tending to one. Note that this bound will pertain to the region $R_{1}$, as $\beta$ is not involved. The bounding function $\bar{C}$ will be shown to be convex. We also will show

$$
\mathcal{L}_{N}(\theta, \widetilde{\beta})-\mathcal{L}_{N}(\widetilde{\theta}, \widetilde{\beta}) \leq \underline{C}(\theta)
$$

for all $\theta \in N_{\delta}(\widetilde{\theta})$, for a convex function $\underline{C}$ and another radius $0<\delta<\epsilon$. It must be chosen such that $\delta^{2} \bar{\Lambda}<\epsilon^{2} \underline{\Lambda}$. In the above error decomposition $\epsilon>0$ was arbitrary, but it will be chosen sufficiently small so as to guarantee that

$$
\sup _{\theta \in N_{\delta}(\widetilde{\theta})} \underline{C}(\theta)+\bar{\Lambda} \delta^{2}<\inf _{\theta \in N_{\epsilon}^{c}(\widetilde{\theta})} \bar{C}(\theta) .
$$

The proofs of (B.11) and (B.12) follow the strategy in Theorem 3.1 of Dahlhaus [3], although we have recourse to Lemma 4.1 for the spatial random field case. The expectation of $\mathcal{L}_{N}(\theta, \widetilde{\beta})-\mathcal{L}_{N}(\widetilde{\theta}, \widetilde{\beta})$ equals

$$
-N^{-2} \log \left|\Sigma^{-1}\left(F_{\theta}\right) \Sigma\left(F_{\widetilde{\theta}}\right)\right|+N^{-2} \operatorname{tr}\left(\Sigma^{-1}\left(F_{\theta}\right) \Sigma\left(F_{\widetilde{\theta}}\right)-1_{N^{2}}\right) .
$$

Here $1_{N^{2}}$ is the identity matrix. Using a Taylor Series expansion, as in Theorem 3.1 of Dahlhaus [3], produces the expectation being equal to

$$
\operatorname{tr}\left(\left(1_{N^{2}}+\tau A\right)^{-1} A\left(1_{N^{2}}+\tau A\right)^{-1} A\right)
$$

where $A=\Sigma^{-1}\left(F_{\theta}\right) \Sigma\left(F_{\widetilde{\theta}}\right)-1_{N^{2}}$ and $\tau$ is some number between 0 and 1 ; note that $\tau$ will depend upon the entries of $A$. The above trace expression can be re-written as a sum of traces of blockToeplitz matrices, to which Lemma 4.1 can be applied. Observing that $A=\Sigma^{-1}\left(F_{\theta}\right)\left[\Sigma\left(F_{\widetilde{\theta}}\right)-\Sigma\left(F_{\theta}\right)\right]$ and $1_{N^{2}}+\tau A=\Sigma^{-1}\left(F_{\theta}\right)\left[(1-\tau) \Sigma\left(F_{\theta}\right)+\tau \Sigma\left(F_{\widetilde{\theta}}\right)\right]$, then $\left(1_{N^{2}}+\tau A\right)^{-1}=\Sigma^{-1}(G) \Sigma\left(F_{\theta}\right)$ with $G=$ $(1-\tau) F_{\theta}+\tau F_{\widetilde{\theta}}$. The sum of the block-Toeplitz matrices is again the block-Toeplitz matrix of $G$, because the summands are positive functions. The dependency of $G$ on $\tau, \theta$, and $\widetilde{\theta}$ will be suppressed in the notation. The trace expression (B.14) can then be expanded using these derivations, which yields the trace of

$$
\begin{aligned}
& \Sigma^{-1}(G) \Sigma\left(F_{\widetilde{\theta}}\right) \Sigma^{-1}(G) \Sigma\left(F_{\widetilde{\theta}}\right) \\
& -\Sigma^{-1}(G) \Sigma\left(F_{\theta}\right) \Sigma^{-1}(G) \Sigma\left(F_{\widetilde{\theta}}\right) \\
& -\Sigma^{-1}(G) \Sigma\left(F_{\widetilde{\theta}}\right) \Sigma^{-1}(G) \Sigma\left(F_{\theta}\right) \\
& +\Sigma^{-1}(G) \Sigma\left(F_{\theta}\right) \Sigma^{-1}(G) \Sigma\left(F_{\theta}\right) .
\end{aligned}
$$


For any fixed $\tau$, the trace of the above expression, divided by $N^{2}$, converges to $\left\langle G^{-2}\left(F_{\widetilde{\theta}}-F_{\theta}\right)^{2}\right\rangle$ as $N_{*} \rightarrow \infty$; therefore the infimum of this quantity over $\tau \in[0,1]$ yields a lower bound, which is a function of $\theta$. Furthermore, utilizing the bound $0<F_{\widetilde{\theta}} / F_{\theta}<K$ for some constant $K$, for all frequencies, we have $G / F_{\theta}<K$ uniformly (and $\tau$ becomes irrelevant). Then let

$$
\bar{C}(\theta)=K^{-2}\left\langle\left(F_{\widetilde{\theta}} / F_{\theta}-1\right)^{2}\right\rangle .
$$

So $\bar{C}(\theta)$ is clearly non-negative, and is zero only when $F_{\widetilde{\theta}}=F_{\theta}$. By the identifiability restriction, this will only occur when $\theta=\widetilde{\theta}$ (the cepstral model class satisfies this condition, due to the basis expansion of log spectra implicit in the model's construction). $\bar{C}(\theta)$ is also concave down, since $x \mapsto(x-1)^{2}$ is [cf., Proposition 2.15, 17].

Moreover, the variance of $\mathcal{L}_{N}(\theta, \widetilde{\beta})-\mathcal{L}_{N}(\widetilde{\theta}, \widetilde{\beta})$ equals $2 N^{-4} \operatorname{tr}\left[A^{2}\right]$ under a Gaussian DGP, and again by Lemma 4.1 - after an expansion of $A^{2}$ - we obtain $O\left(N^{-2}\right)$ growth. This establishes (B.11) for any $\theta$. By taking the infimum of $\bar{C}(\theta)$ over $N_{\epsilon}^{c}(\widetilde{\theta})$, we can ensure a non-zero value as well, utilizing the convexity of the function. For the other bound, note that for any $\theta_{1}, \theta_{2}$ we have

$$
\mathcal{L}_{N}\left(\theta_{2}, \widetilde{\beta}\right)-\mathcal{L}_{N}\left(\theta_{1}, \widetilde{\beta}\right)=N^{-2}\left(\theta_{2}-\theta_{1}\right)^{\prime} \operatorname{tr}\left\{\Sigma^{-1}\left(F_{\theta}\right) \Sigma\left(\nabla F_{\theta}\right)\right\}+N^{-2} W^{\prime}\left(\Sigma^{-1}\left(F_{\theta_{2}}\right)-\Sigma^{-1}\left(F_{\theta_{1}}\right)\right) W,
$$

where $\theta$ is in-between $\theta_{1}$ and $\theta_{2}$, using the Mean Value Theorem and the assumed smoothness on spectra. The notation $\Sigma\left(\nabla F_{\theta}\right)$ denotes an array of matrices, indexed by the various derivatives of $F_{\theta}$; after the trace has been computed, the resulting vector is multiplied by $\left[\theta_{2}-\theta_{1}\right]^{\prime}$. Using the smoothness conditions on spectra, we may apply Lemma 5.5 of Dahlhaus [3] and our Lemma 4.1 to bound the absolute value of the above difference by a constant times $\left\|\theta_{2}-\theta_{1}\right\|$, with probability tending to one. Letting $\theta_{1}$ be $\widetilde{\theta}$ and $\underline{C}(\theta)=K\|\theta-\widetilde{\theta}\|$ for some constant $K>0$ now yields (B.12).

Because of the condition on $M(\theta)$, its eigenvalues are uniformly (in $\theta$ ) bounded between $0<\underline{\Lambda}$ and $\bar{\Lambda}<\infty$, and the quadratic form satisfies

$$
Q(\theta, \beta) \geq \underline{\Lambda} \epsilon^{2}
$$

for all $\beta \in N_{\epsilon}^{c}(\widetilde{\beta})$ and any $\theta$. Furthermore we also have

$$
Q(\theta, \beta) \leq \bar{\Lambda} \delta^{2}
$$

for all $\beta \in N_{\delta}(\widetilde{\beta})$ and any $\theta$. Below, it is shown that we must select $\delta$ for a given $\epsilon$ such that $\delta^{2} \bar{\Lambda}<\epsilon^{2} \underline{\Lambda}$. Next, using the convexity of $\underline{C}$ and $\bar{C}$, adjusting their constants if necessary, we can ensure that (B.13) holds for some $\epsilon$, making $\delta$ smaller if necessary. Now the second term in the parameter error can be rewritten as

$$
\frac{\int_{\bar{R}_{1} \cup \bar{R}_{2} \bigcup\left(R_{1} \cap R_{2}\right)}(\phi-\widetilde{\phi}) \exp \left\{-\frac{N^{2}}{2}\left[\mathcal{L}_{N}(\theta, \widetilde{\beta})-\mathcal{L}_{N}(\widetilde{\theta}, \widetilde{\beta})+E_{N}+Q(\theta, \beta)\right]\right\} p(\phi) d \phi}{\int \exp \left\{-\frac{N^{2}}{2}\left[\mathcal{L}_{N}(\theta, \widetilde{\beta})-\mathcal{L}_{N}(\widetilde{\theta}, \widetilde{\beta})+E_{N}+Q(\theta, \beta)\right]\right\} p(\phi) d \phi}
$$

by multiplying top and bottom by the constant $\mathcal{L}_{N}(\widetilde{\theta}, \widetilde{\beta})$ and utilizing (B.10). Furthermore, the denominator (which is a scalar) can be made smaller by restricting the region of integration, which only increases the absolute error of each component of the parameter error vector. We will restrict the integration to the set $N_{\delta}(\widetilde{\theta}) \times N_{\delta}(\widetilde{\beta})$. At this point, we break the analysis up by considering the numerator integral (still as a vector quantity) over the various disjoint regions. We present an analysis for integration over $R_{1}$ and $R_{2}$, and note that either analysis will then pertain to $R_{1} \bigcap R_{2}$. 
First consider the numerator restricted to integration over $R_{1}$, and take the Euclidean norm of this portion of the error vector; then we obtain the upper bound

$$
\begin{aligned}
& \frac{\int_{R_{1}}\|\phi-\widetilde{\phi}\| \exp \left\{-\frac{N^{2}}{2}[\bar{C}(\theta)]\right\} p(\phi) d \phi}{\int_{N_{\delta}(\widetilde{\theta}) \times N_{\delta}(\widetilde{\beta})} \exp \left\{-\frac{N^{2}}{2}\left[\underline{C}(\theta)+\underline{\Lambda} \delta^{2}\right]\right\} p(\phi) d \phi} \\
& \leq \frac{\int_{R_{1}}\|\phi-\widetilde{\phi}\| \exp \left\{-\frac{N^{2}}{2}\left[\inf _{\theta \in N_{\epsilon}^{c}(\widetilde{\theta})} \bar{C}(\theta)-\sup _{\theta \in N_{\delta}(\widetilde{\theta})} \underline{C}(\theta)-\bar{\Lambda} \delta^{2}\right]\right\} p(\phi) d \phi}{\int_{N_{\delta}(\widetilde{\theta}) \times N_{\delta}(\widetilde{\beta})} p(\phi) d \phi}
\end{aligned}
$$

with probability tending to one. The second expression follows from the first by taking supremums and infinums. The first expression uses the triangle inequality for integrals along with (B.11), (B.12), and (B.16). Note that these bounds hold with probability tending to one, and therefore they also hold with probability tending to one when the term $E_{N}$ (which tends to zero in probability as $N_{*} \rightarrow \infty$ ) is inserted. In the numerator, (B.11) applies because we are in the set $R_{1}$, whereas the other bounds hold on the set $N_{\delta}(\widetilde{\theta}) \times N_{\delta}(\widetilde{\beta})$. The overall bound on this term tends to zero as $N_{*} \rightarrow \infty$, because the numerator integral involves the exponential of $-N^{2}$ times a positive quantity - it is positive by (B.13).

Next, we consider the numerator restricted to $R_{2}$, and mimic the same line of argument, obtaining the upper bound

$$
\begin{aligned}
& \frac{\int_{R_{2}}\|\phi-\widetilde{\phi}\| \exp \left\{-\frac{N^{2}}{2}\left[\underline{\Lambda} \epsilon^{2}\right]\right\} p(\phi) d \phi}{\int_{N_{\delta}(\widetilde{\theta}) \times N_{\delta}(\widetilde{\beta})} \exp \left\{-\frac{N^{2}}{2}\left[\underline{C}(\theta)+\underline{\Lambda} \delta^{2}\right]\right\} p(\phi) d \phi} \\
& \leq \frac{\int_{R_{2}}\|\phi-\widetilde{\phi}\| \exp \left\{-\frac{N^{2}}{2}\left[\underline{\Lambda} \epsilon^{2}-\sup _{\theta \in N_{\delta}(\widetilde{\theta})} \underline{C}(\theta)-\bar{\Lambda} \delta^{2}\right]\right\} p(\phi) d \phi}{\int_{N_{\delta}(\widetilde{\theta}) \times N_{\delta}(\widetilde{\beta})} p(\phi) d \phi}
\end{aligned}
$$

with probability tending to one, using (B.15). Now we need the condition that $\sup _{\theta \in N_{\delta}(\tilde{\theta})} \underline{C}(\theta)<$ $\epsilon^{2} \underline{\Lambda}-\delta^{2} \bar{\Lambda}$, which is assured by taking $\delta$ even smaller as needed. Then this term also tends to zero as $N_{*} \rightarrow \infty$, which concludes the proof.

\section{References.}

[1] Brillinger, D. R. (2001). Time Series: Data Analysis and Theory 36. Philadelphia: SIAM.

[2] Cressie, N. (1993). Statistics for Spatial Data. New York: John Wiley and Sons.

[3] Dahlhaus, R. (1989). Efficient Parameter Estimation for Self-Similar Processes. The Annals of Statistics 17 1749-1766.

[4] Dahlhaus, R. and Wefelmeyer, W. (1996). Asymptotically Optimal Estimation in Misspecified Time Series Models. The Annals of Statistics 24 952-974.

[5] Guyon, X. (1982). Parameter Estimation for a Stationary Process on a d-Dimensional Lattice. Biometrika 69 95-105.

[6] Hodges, J. S. and Reich, B. J. (2010). Adding Spatially-Correlated Errors Can Mess Up the Fixed Effect You Love. The American Statistician 64 325-334.

[7] Holan, S., McElroy, T. and Chakraborty, S. (2009). A Bayesian Approach to Estimating the Long Memory Parameter. Bayesian Analysis 4 159-190.

[8] KizilkayA, A. (2007). On the Parameter Estimation of 2-D Moving Average Random Fields. Circuits and Systems II: Express Briefs, IEEE Transactions on 54 989-993.

[9] Liseo, B., Marinucci, D. and Petrella, L. (2001). Bayesian Semiparametric Inference on Long-Range Dependence. Biometrika 88 1089-1104.

[10] Mardia, K. and Marshall, R. (1984). Maximum Likelihood Estimation of Models for Residual Covariance in Spatial Regression. Biometrika 71 135-146.

[11] McElroy, T. S. and Holan, S. H. (2009). A Local Spectral Approach for Assessing Time Series Model Misspecification. Journal of Multivariate Analysis 100 604-621. 
[12] Mercer, W. and Hall, A. (1911). The Experimental Error of Field Trials. Journal of Agricultural Science 4 107-132.

[13] Paciorek, C. J. (2010). The Importance of Scale for Spatial-Confounding Bias and Precision of Spatial Regression Estimators. Statistical Science 25 107-125.

[14] Solo, V. (1986). Modeling of Two-Dimensional Random Fields by Parametric Cepstrum. IEEE Transactions on Information Theory 32 743-750.

[15] Taniguchi, M. and Kakizawa, Y. (2000). Asymptotic Theory of Statistical Inference for Time Series. New York: Springer.

[16] R Development Core Team (2012). R: A Language and Environment for Statistical Computing R Foundation for Statistical Computing, Vienna, Austria ISBN 3-900051-07-0.

[17] VAJDA, I. (1989). Theory of Statistical Inference and Information. Kluwer Academic Publishers Boston.

[18] Wahba, G. (1968). On the Distribution of Some Statistics Useful in the Analysis of Jointly Stationary Time Series. The Annals of Mathematical Statistics 1849-1862. 
TABLE 1

Log likelihood $(\log (L))$, Akaike information criterion (AIC), Bayesian information criterion (BIC), and Hannan-Quinn information criterion (HQ) for the models evaluated in Section A.2. T and NT denote models with the trend (row and column) effect accounted for and no-trend effect accounted for (i.e., constant-mean only), respectively. The bold entries are the minimum $A I C, B I C$ and $H Q$.

\begin{tabular}{|l|r|r|r|}
\hline & $p=1$ & $p=2$ & $p=3$ \\
\hline$-\log (L)_{T}$ & 64.906 & 36.985 & 21.195 \\
$-\log (L)_{N T}$ & 85.245 & 51.113 & 30.499 \\
$\mathrm{AIC}_{T}$ & 145.813 & 105.970 & $\mathbf{9 8 . 3 8 8}$ \\
$\mathrm{AIC}_{N T}$ & 182.490 & 130.226 & 112.998 \\
$\mathrm{BIC}_{T}$ & 179.530 & $\mathbf{1 7 3 . 4 0 4}$ & 216.399 \\
$\mathrm{BIC}_{N T}$ & 207.778 & 189.230 & 222.578 \\
$\mathrm{HQ}_{T}$ & 159.043 & $\mathbf{1 3 2 . 4 3 1}$ & 144.696 \\
$\mathrm{HQ}_{N T}$ & 192.413 & 153.379 & 155.997 \\
\hline
\end{tabular}

TABLE 2

Straw yield data analysis results (Section A.2). Note that the estimated standard error is obtained as the square root of the diagonal of the inverse observed Hessian matrix and that elements of $\theta$ are described in Section 5 of the article.

\begin{tabular}{|c|r|r|}
\hline Parameter & Estimate & Standard Error \\
\hline$\theta_{1}$ & 0.009 & 0.052 \\
$\theta_{2}$ & -0.028 & 0.049 \\
$\theta_{3}$ & 0.132 & 0.052 \\
$\theta_{4}$ & 0.067 & 0.049 \\
$\theta_{5}$ & 0.271 & 0.047 \\
$\theta_{6}$ & 0.383 & 0.046 \\
$\theta_{7}$ & 0.001 & 0.055 \\
$\theta_{8}$ & -0.017 & 0.048 \\
$\theta_{9}$ & -0.003 & 0.051 \\
$\theta_{10}$ & -0.055 & 0.049 \\
$\theta_{11}$ & -0.015 & 0.047 \\
$\theta_{12}$ & 0.144 & 0.047 \\
$\theta_{13}$ & -0.871 & 0.063 \\
$\beta_{0}$ & 7.646 & 0.176 \\
$\beta_{1}$ & -0.035 & 0.010 \\
$\beta_{2}$ & -0.059 & 0.009 \\
\hline
\end{tabular}




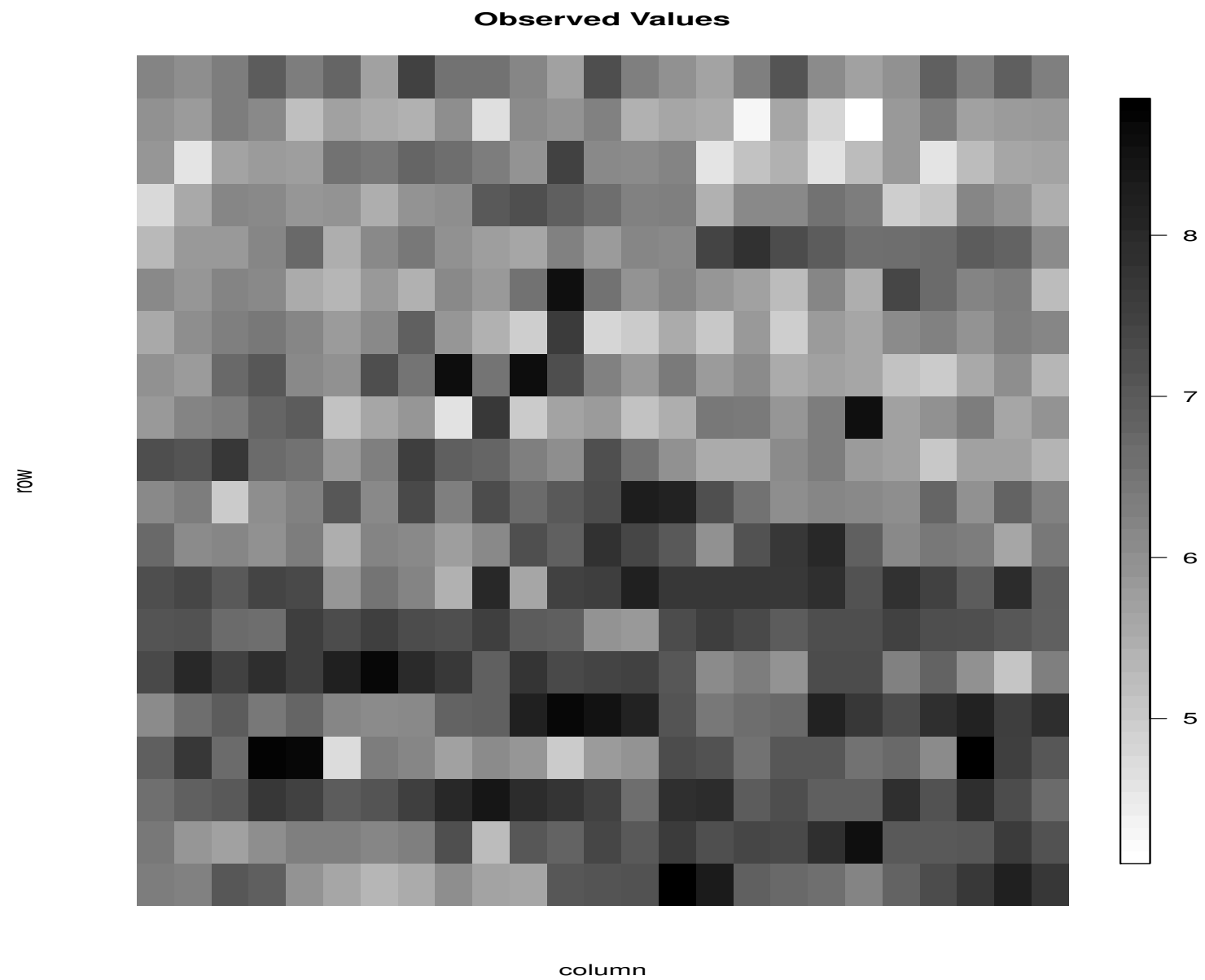

FIg 1. Observed Mercer and Hall straw yield data [12]. The observed lattice is $20 \times 25$. Full details of the agricultural experiment are described in Section A.2.

Center for Statistical Research and Methodology, U.S. Census Bureau, 4600 Silver Hill Road,

WASHington, D.C. 20233-9100

E-MAIL: tucker.s.mcelroy@census.gov
Department of Statistics,

University of Missouri, 146 Middlebush Hall, Columbia, MO, 65211-6100

E-MAIL: holans@missouri.edu 